\title{
IN VITRO - IN VIVO CORRELATION FOR GLICLAZIDE 60 MG MODIFIED RELEASE TABLETS
}

\section{DIANA IOANA POP ${ }^{a, b}$, ADRIANA MARCOVICI ${ }^{b}$, MONICA OROIAN ${ }^{a, b}$, ANA-MARIA GHELDIU ${ }^{*}$, LAURIAN VLASE ${ }^{a}$}

\begin{abstract}
The objective of the study was to determine in vitro - in vivo correlations for Gliclazide $60 \mathrm{mg}$ modified release tablets developed by Ranbaxy Laboratories Limited, now Sun Pharmaceutical Industries Limited, India, based on the data obtained in two bioequivalence clinical trials and in in vitro dissolution tests. Each clinical trial was designed as an open-label, randomized, single-dose, crossover study that consisted of two periods. The first bioequivalence study was performed under the fasting state of the subjects, while the second bioequivalence study was carried out under the fed state of the subjects. During each study period, venous blood samples were taken pre-dose and post-dose up to 96 hours. Afterwards, individual plasma profiles were obtained and mathematical deconvolution was applied to obtain the relative fraction absorbed of gliclazide. These data were correlated with the in vitro dissolution data obtained after performing dissolution tests in three different dissolution media, at $\mathrm{pH} 4.5,6.8$ and 7.2, with Gliclazide $60 \mathrm{mg}$ modified release tablets (EvoluPharm, France). All calculation were performed by Phoenix WinNonlin ${ }^{\circledR}$ version 6.3. For each in vivo data set from the bioequivalence studies (under fasting and fed conditions), three level $\mathrm{A}$ in vitro-in vivo correlations were obtained for Gliclazide $60 \mathrm{mg}$ modified release tablets, for in vitro tests performed at $\mathrm{pH} 4.5,6.8$, and 7.2. Good correlation coefficients were found for each established correlation (R2=0.98-0.99). In
\end{abstract}

a University of Medicine and Pharmacy 'Iuliu Hatieganu', Faculty of Pharmacy, Department of Pharmaceutical Technology and Biopharmaceutics, 8 Victor Babes str., RO-400012, ClujNapoca, Romania

b Terapia SA - a Sun Pharma Company, Department of Clinical Pharmacology and Pharmacokinetics, 124 Fabricii str., RO-400632, Cluj-Napoca, Romania

c University of Medicine and Pharmacy 'Iuliu Hatieganu', Faculty of Pharmacy, Department of Pharmaceutical Botany, 23 Marinescu str., RO-400337, Cluj-Napoca, Romania

*Corresponding author: anamaria.gheldiu@yahoo.com 
conclusion, six level A in vitro-in vivo correlations were obtained for Gliclazide $60 \mathrm{mg}$ modified release tablets manufactured by Ranbaxy Laboratories Limited, now Sun Pharmaceutical Industries Limited, India.

Keywords: gliclazide, in vitro-in vivo correlations, clinical trial, healthy Caucasian subjects

\section{INTRODUCTION}

The oral absorption, and therefore the bioavailability of a drug, is determined by the extent of drug aqueous solubility and permeability along the gastro-intestinal tract. The Biopharmaceutical Classification System (BCS) is the guiding tool used for the prediction of a drug's in vivo performance and development of drug delivery system that suits this performance [1]. It consists of four classes, which categorizes the drug substances based on the previously mentioned properties as it follows: class I - high solubility and high permeability, class II - low solubility and high permeability, class III - high solubility and low permeability, class IV - low solubility and low permeability [1]. Because of its application in early drug development and afterwards in the management of product post-approval changes, the interest in this BCS is high [2].

Bioequivalent drug products are pharmaceutical equivalents or pharmaceutical alternatives whose rate and extent of absorption are comparable, without statistically significant differences, when administered at the same molar dose of the therapeutic moiety under similar experimental conditions, either single dose or multiple dose [3]. Bioequivalence clinical studies conducted on healthy volunteers are requested for pharmaceutically equivalent drugs (generics), but which may be different in terms of the nature and quantity of excipients and manufacturing process [4]. In the last years, different approaches were developed to reduce the need for in vivo bioequivalence studies, considering that the costs for such trials are high $[4,5]$. Regulatory authorities adopted several guidelines on biowaivers that could be accepted by application of the BCS based scheme or by establishing an in vitro-in vivo correlation (IVIVC) $[4,6,7,8]$. A biowaiver represents the permission given by the regulatory authorities to use dissolution test as a surrogate of pharmacokinetic data obtained in bioequivalence studies, in cases when scale-up and post-approval (SUPAC) related changes occur.

An in vitro-in vivo correlation (IVIVC) was defined by the U.S. Food and Drug Administration (FDA) as "a predictive mathematical model describing the relationship between an in-vitro property of a dosage form and an in-vivo response" [7]. In addition, IVIVCs are also defined in the United States Pharmacopoeia (USP) [16]. In vitro property refers to dissolution rate, dissolution profile, mean dissolution time (MDT), and percent dissolved at a 
certain time point $\left(t_{x} \%\right)$ [3]. In vivo response is the plasma drug profile, the amount absorbed at a certain time ( $\left.Q_{a b s}\right)$, the mean absorption time (MAT), or other pharmacokinetic (PK) parameters such as peak plasma concentration $\left(\mathrm{C}_{\max }\right)$ or observed area under the plasma concentration versus time curve $\left(A \cup C_{0-t}\right)[3]$. Currently, four distinct IVIVC levels were established: level A, level $\mathrm{B}$, level $\mathrm{C}$ and multiple level $\mathrm{C}$. Level $\mathrm{A}$ is a point to point correlation between in vitro dissolution and in vivo absorption rate of a drug from the dosage form $[3,7,16]$. Level A IVIVC is of highest regulatory value as its purpose is to define a direct relationship such that measurement of in vitro dissolution rate is a surrogate for in vivo performance. Hence, this level of IVIVC is an excellent quality control procedure since it is predictive of the dosage forms in vivo performance and can be used to apply for a biowaiver [3]. Usually, biowaiver is not possible for level $B, C$ and multiple $C[3,4,7,9]$.

Currently, the IVIVC based biowaiver is recommended by regulatory authorities for modified release dosage forms with extended release and recently it was suggested that this type of waiver could be also applied to Class II BCS drugs, as long as the dissolution process is complete during the gastro-intestinal ( $\mathrm{GI}$ ) passage $[4,6,7,8,10]$. In case of BCS class II drugs, an IVIVC is expected if in vitro dissolution rate is similar to in vivo dissolution rate, which is correlated with in vivo absorption rate [1].

Gliclazide (see Figure 1) is an oral hypoglycemic agent for the treatment of type 2 diabetes mellitus (T2DM), which belongs to BCS Class II. It presents high permeability and an intermediate solubility, as it is an ampholyte which solubility is $\mathrm{pH}$-dependent in the $\mathrm{Gl} \mathrm{pH}$ range. After oral administration, it was reported that gliclazide displays a non-solubility-limited absorption. The absorption rate and the onset of action is delayed after oral administration $[6,11,12,13]$. Gliclazide has a wide therapeutic index and displays linear profile for doses up to $120 \mathrm{mg}[19,20]$. Although its presystemic metabolism has not been thoroughly studied, the low plasma clearance of $13 \mathrm{~mL} / \mathrm{min}(0.78 \mathrm{~L} / \mathrm{h})$ suggests this elimination pathway is not significant [18]. Considering the pharmacological and pharmaceutical profile, gliclazide is eligible for assessment of level A IVIVC.

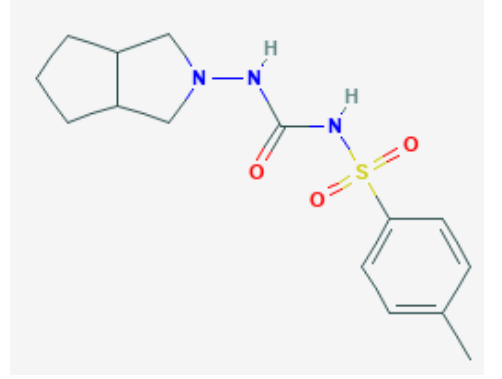

Figure 1. Chemical structure of gliclazide (IUPAC name: 1-(3,3a,4,5,6,6ahexahydro-1H-cyclopenta[c]pyrrol-2-yl)-3-(4-methylphenyl)sulfonylurea) [22] 
For establishing level A IVIVC, the most common process is to develop formulations with different release rates (slow, medium and fast) and then to obtain for these formulation in vitro dissolution profiles and in vivo plasma concentration profiles [7]. Afterwards, an appropriate deconvolution method is applied to each formulation in order to estimate the in vivo absorption [7]. The purpose of developing such an IVIVC is to prove that in vitro dissolution characteristics are predictable for in vivo performance of a drug product and are maintained over a range of in vitro dissolution release rates, used to define the IVIVC relationship and manufacturing changes [7].

The aim of this study was to evaluate the possibility of establishing level A IVIVC for gliclazide $60 \mathrm{mg}$ modified release tablets from in vitro studies at different $\mathrm{pH}(4.5,6.8$ and 7.2$)$ with in vivo bioequivalence studies, under fasting and fed conditions of subjects. The current research is a preliminary study whose objective was not to develop an industrial IVIVC, but to support additional studies to this purpose. Therefore, the in vitro studies were performed for a single modified release formulation of gliclazide.

\section{RESULTS AND DISCUSSION}

\section{In vivo data}

The in vivo data that were used for determination of IVIVC for gliclazide were obtained by mathematical deconvolution. This approach considers the available output function, the mean plasma concentrations, for determining the input function, the absorption results of gliclazide. Thus, the relative fraction absorbed of gliclazide from the site of administration over time was obtained and is depicted in Figure 2. The data of the bioequivalence studies were previously reported $[11,14,15]$.

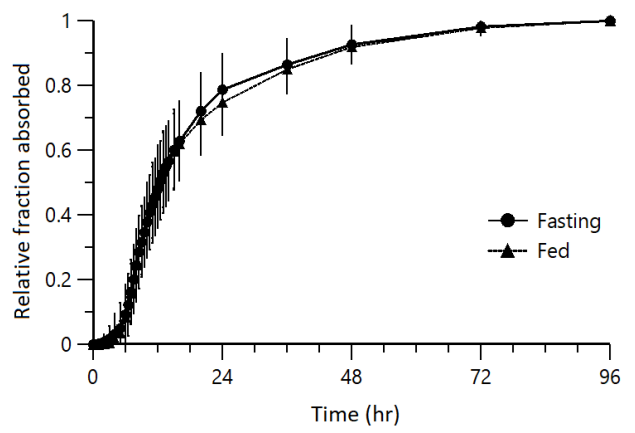

Figure 2. The relative fraction absorbed of gliclazide (60 mg, p.o.) in the systemic circulation from the site of administration over time in subjects under fasting $(n=41)$ or fed conditions $(n=23)$ [11] 


\section{In vitro studies}

By using the methodology and sample preparation described in the experimental section, the percent of dissolved gliclazide from the $60 \mathrm{mg}$ modified release generic tablets were obtained, in three distinct dissolution media. The first in vitro test was performed in $\mathrm{pH} 4.5$ sodium acetate buffer, the second test was carried out in $\mathrm{pH} 6.8$ phosphate buffer and the third in vitro dissolution test was realized in $\mathrm{pH} 7.2$ phosphate buffer. The results are summarized in Table 1. The in vitro release profiles over 24 hours of gliclazide $60 \mathrm{mg}$ generic modified release tablets in three different dissolution media are depicted in Figure 3. Data are shown as mean \pm standard deviation $(n=6)$.

Table 1. In vitro dissolution profiles of $60 \mathrm{mg}$ generic gliclazide modified release tablets in three different dissolution media ( $\mathrm{pH} \mathrm{4.5,6.8} \mathrm{and} \mathrm{7.2)}$

\begin{tabular}{|l|l|l|l|l|l|l|l|l|l|}
\hline \multirow{2}{*}{$\begin{array}{c}\text { Time } \\
\text { (hr) }\end{array}$} & \multicolumn{3}{|c|}{ \% Drug release } & \multicolumn{3}{c|}{ pH 6.8 } & \multicolumn{3}{c|}{ pH 7.2 } \\
\cline { 2 - 10 } & Mean $^{1}$ & SD $^{2}$ & CV\% $^{3}$ & Mean $^{1}$ & SD $^{2}$ & CV\% $^{3}$ & Mean $^{1}$ & SD $^{2}$ & CV\% $^{3}$ \\
\hline 0.5 & 0.8 & 1.3 & 173.2 & 1.8 & 0.2 & 12.9 & 2.6 & 0.5 & 18.1 \\
\hline 1 & 3.9 & 0.5 & 13.9 & 4.8 & 1.4 & 29.4 & 5.3 & 0.1 & 2.6 \\
\hline 1.5 & 6.7 & 3.1 & 46.3 & 5.6 & 0.2 & 3.2 & 8.2 & 0.9 & 11.1 \\
\hline 2 & 9.5 & 3.3 & 34.2 & 8.6 & 1.6 & 18.4 & 11.2 & 1.5 & 13.6 \\
\hline 2.5 & 14.6 & 0.9 & 6.3 & 10.9 & 1.8 & 17.0 & 13.5 & 0.7 & 5.3 \\
\hline 3 & 18.2 & 1.2 & 6.4 & 13.8 & 1.2 & 8.7 & 16.8 & 0.8 & 4.8 \\
\hline 3.5 & 19.7 & 2.0 & 10.3 & 15.4 & 1.6 & 10.2 & 19.2 & 0.5 & 2.5 \\
\hline 4 & 22.7 & 2.8 & 12.2 & 18.1 & 1.7 & 9.3 & 21.8 & 1.7 & 7.8 \\
\hline 5 & 26.3 & 4.6 & 17.5 & 22.8 & 2.9 & 12.7 & 27.2 & 3.3 & 12.2 \\
\hline 6 & 30.5 & 2.5 & 8.2 & 28.6 & 2.2 & 7.6 & 33.6 & 1.3 & 3.7 \\
\hline 7 & 35.5 & 1.5 & 4.3 & 33.7 & 2.6 & 7.7 & 37.8 & 2.8 & 7.3 \\
\hline 8 & 41.9 & 1.3 & 3.2 & 38.3 & 3.0 & 7.9 & 42.8 & 2.6 & 6.1 \\
\hline 9 & 45.9 & 0.6 & 1.4 & 43.9 & 2.4 & 5.4 & 50.2 & 3.8 & 7.5 \\
\hline 10 & 49.7 & 2.1 & 4.2 & 49.6 & 4.8 & 9.6 & 54.3 & 3.4 & 6.2 \\
\hline 12 & 55.7 & 1.4 & 2.6 & 59.2 & 4.4 & 7.3 & 64.7 & 5.1 & 7.9 \\
\hline 14 & 60.4 & 2.0 & 3.3 & 67.4 & 4.0 & 5.9 & 73.7 & 5.8 & 7.9 \\
\hline 16 & 63.4 & 0.3 & 0.4 & 76.2 & 3.1 & 4.0 & 80.5 & 5.8 & 7.2 \\
\hline 24 & 69.3 & 3.7 & 5.3 & 94.2 & 2.5 & 2.6 & 94.1 & 3.2 & 3.4 \\
\hline
\end{tabular}

${ }^{1}$ Data are shown as mean of $n=6 ;{ }^{2} \mathrm{SD}$ - standard deviation; ${ }^{3} \mathrm{CV} \%$ - coefficient of variation 


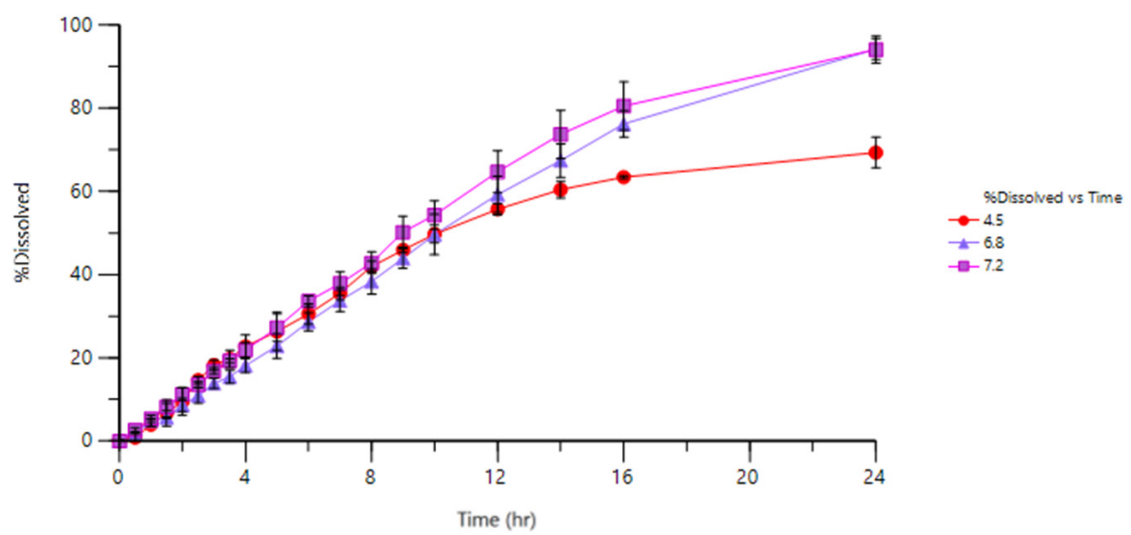

Figure 3. The 24 hours in vitro release profiles of Gliclazide $60 \mathrm{mg}$ generic modified release tablets in various dissolution media with different $\mathrm{pH}$ (data are shown as mean $\pm S D, n=6$ )

\section{In vitro - in vivo correlation}

The in vitro-in vivo correlations (IVIVCs) were evaluated for level $\mathrm{A}$ correlation. Hence, the absorption profiles of Gliclazide $60 \mathrm{mg}$ modified release tablets manufactured by Ranbaxy Laboratories Limited, now Sun Pharmaceutical Industries Limited, India, were obtained from the individual plasma drug concentration versus time profiles from the bioequivalence trials $[11,14,15]$. The absorption profiles were determined for both clinical trials performed under fasting or fed conditions. Therefore, the percent of in vivo absorbed gliclazide was correlated with the in vitro dissolved percent. Figures 4 and 5 illustrate the IVIVCs without time scaling, while figures 6 and 7 depict the IVIVCs that took into consideration a time scaling factor, which was the observed lag time in the in vivo data, in order to obtain a meaningful IVIVC. The obtained slopes, intercepts, and correlation coefficients of the regression lines for IVIVCs are shown in Table 2.

Table 2. Results of the in vitro-in vivo correlation (IVIVC) of Gliclazide $60 \mathrm{mg}$ modified release tablets and the statistical evaluation of the obtained IVIVC for the fasting and fed clinical trials (deconvolution approach)

\begin{tabular}{|c|c|c|c|c|c|c|}
\hline \multicolumn{3}{|c|}{ Clinical trial type } & \multicolumn{3}{c|}{ Fed } \\
\hline $\begin{array}{c}\text { Dissolution } \\
\text { media pH }\end{array}$ & Slope & Intercept & Correlation & Slope & Intercept & Correlation \\
\hline 4.5 & 0.855 & 4.781 & 0.990 & 0.826 & 1.327 & 0.994 \\
\hline 6.8 & 0.927 & 1.720 & 0.993 & 0.884 & -1.614 & 0.984 \\
\hline 7.2 & 1.002 & 3.329 & 0.993 & 0.959 & -0.401 & 0.988 \\
\hline
\end{tabular}




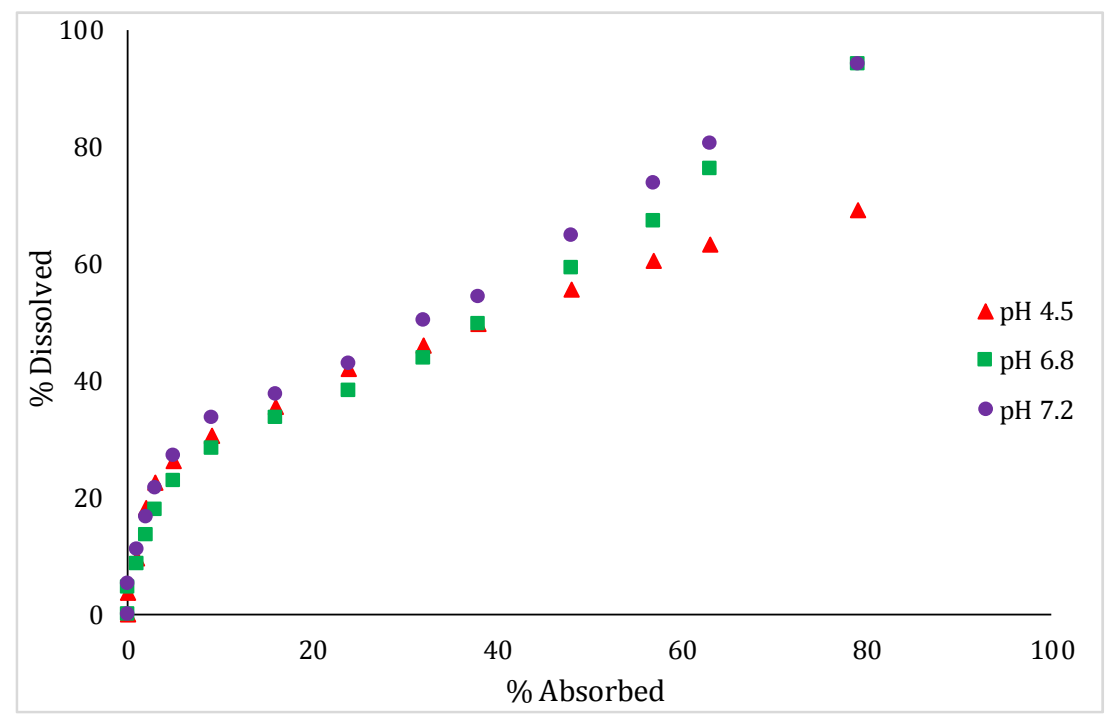

Figure 4. In vitro - in vivo correlation plot for Gliclazide $60 \mathrm{mg}$ modified release tablets (deconvolution approach) at various $\mathrm{pH}$ of the dissolution media of in vitro tests (no time scaling factor), under the fasting state of the in vivo clinical trial

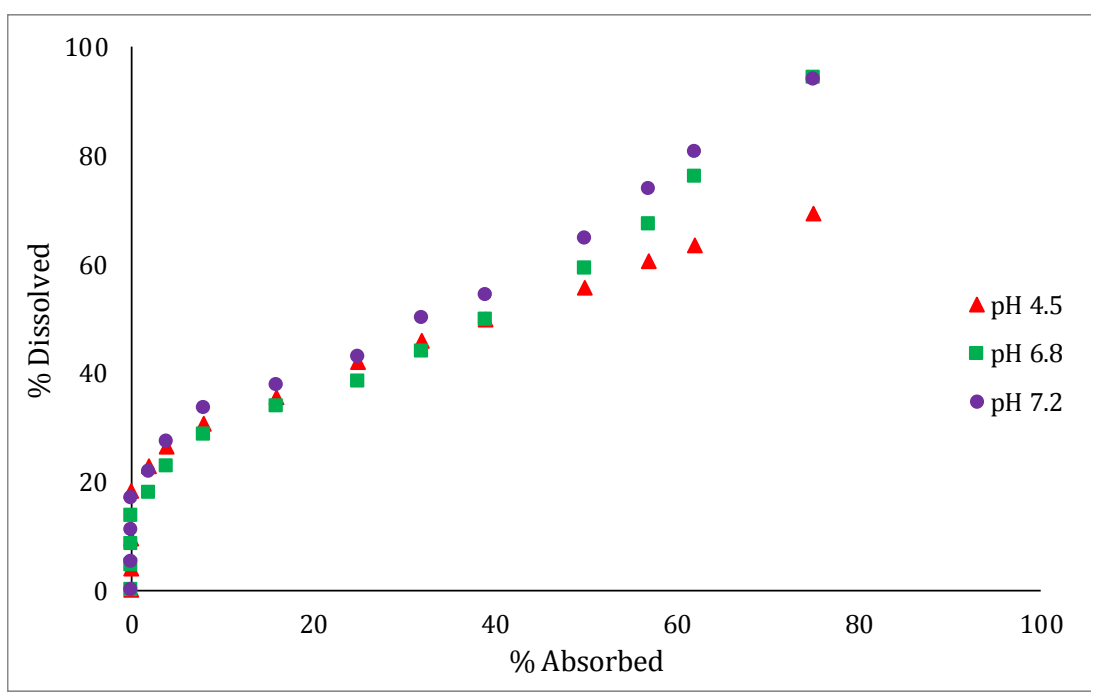

Figure 5. In vitro - in vivo correlation plot for Gliclazide $60 \mathrm{mg}$ modified release tablets (deconvolution approach) at various $\mathrm{pH}$ of the dissolution media of in vitro tests (no time scaling factor), under the fed state of the in vivo clinical trial 


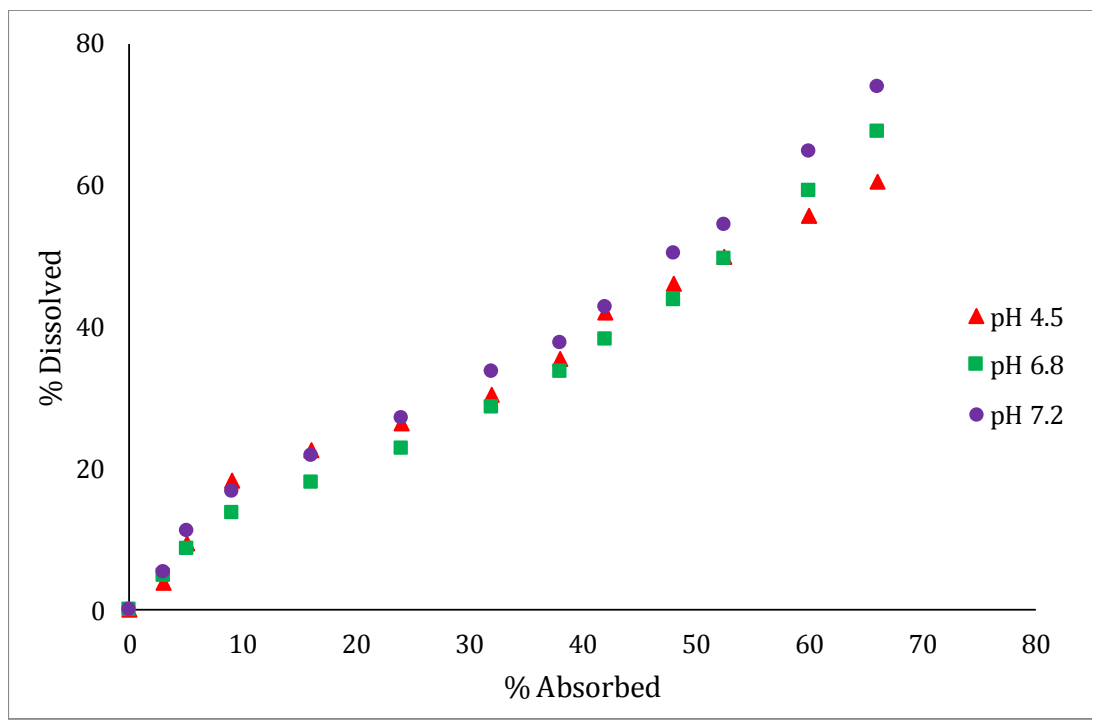

Figure 6. In vitro - in vivo correlation plot for Gliclazide $60 \mathrm{mg}$ modified release tablets (deconvolution approach) at various $\mathrm{pH}$ of the dissolution media of in vitro tests (with time scaling factor), for the fasting state of the subjects of the in vivo clinical trial

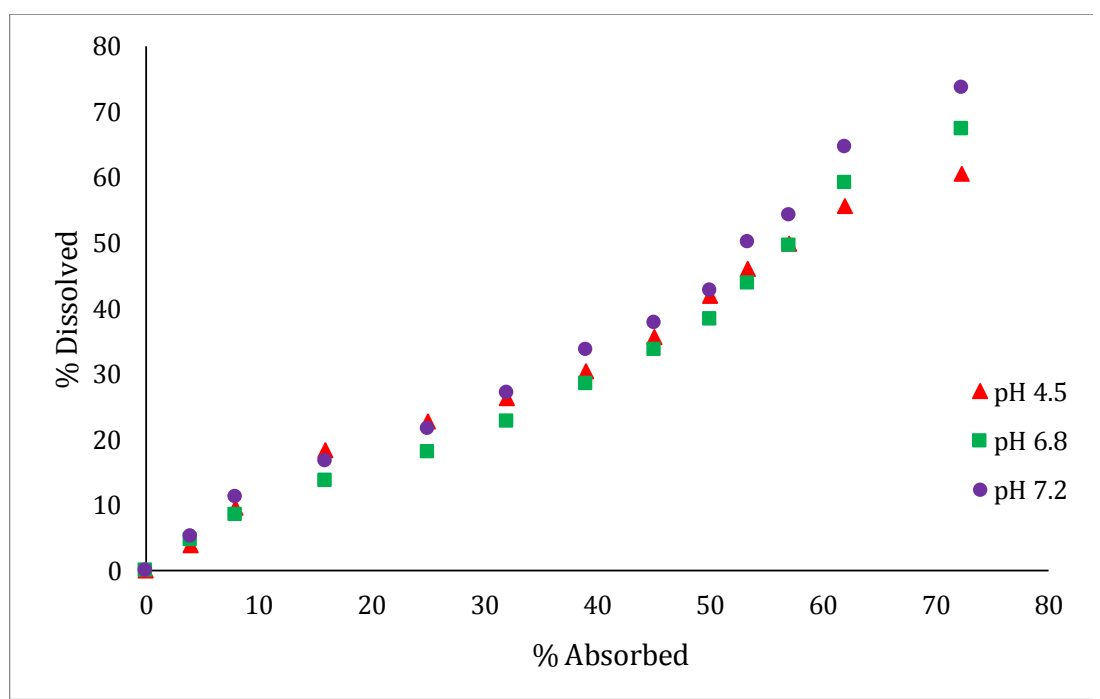

Figure 7. In vitro - in vivo correlation plot for Gliclazide $60 \mathrm{mg}$ modified release tablets (deconvolution approach) at various $\mathrm{pH}$ of the dissolution media of in vitro tests (with time scaling factor), for the fed state of the subjects of the in vivo clinical trial 
In order to establish an IVIVC, the factor controlling the appearance of the active pharmaceutical ingredient (API) in the blood stream should be linked with the formulation or characteristics of the API. In this case, any physiological factor should not be a limiting factor for drug absorption. Thus, IVIVC are probable in practice for API belonging to BCS class II (low solubility, high permeability) in drug dosage forms with slow release [18]. The newly developed generic tablet of gliclazide $60 \mathrm{mg}$ was designed as a modified release (MR) formulation for which the tablet's hydration leads to formation of a gel layer through which the API is slowly released in time $[11,14,15]$. Hence, the drug dosage type controls the absorption of gliclazide and its low solubility is not a rate-limiting step for absorption. Gliclazide MR was shown to have predictable and reproducible release of the API and effective plasma concentrations over a 24 hours' period taken as a single daily dose (30 -120 mg), thus assuring a hypoglycemic effect for a longer time-period $[14,15,19,20]$.

In order to produce a meaningful IVIVC for gliclazide, it was assumed that the in vivo profile and the in vitro profile should not be different in their morphology (shape). In the case of MR tablets, the in vitro dissolution profiles of gliclazide ran ahead the in vivo profiles, which were obtained by using the mathematical deconvolution approach. This was probably due to slow release of the API, additionally to the biphasic gastric emptying. Another possible explanation would be the absorption of gliclazide from two distinct sites within the upper gastrointestinal tract [11]. The time-lapse between the administration of the drug and the start of the absorption process is highlighted by the lag time, which is approximately 3 hours and can be observed in figures 3 and 4 . Thus, the rescaling of the time by considering a time scaling factor of $3 \mathrm{hrs}$ was necessary, this being the latency time observed for in vivo absorption in both clinical trials $[14,15,17]$. Afterwards, the IVIVCs were established by taking into account the in vitro data that corresponded to the in vivo data after rescaling the time axis (time-adjustment), for the dissolution tests performed under different $\mathrm{pH}$ conditions.

The in vitro dissolution profile for the test performed at $\mathrm{pH} 4.5$ revealed a lower release of the drug from the pharmaceutical formulation or instability of gliclazide at acidic $\mathrm{pH}$. [21]. However, for this in vitro profile the IVIVC, a good correlation coefficient was observed, as illustrated by the statistical parameters in Table 2. For the in vitro dissolution profiles obtained by working at $\mathrm{pH}$ of $4.5,6.8$ and 7.2 , a linear correlation was found with the absorption in vivo data. Hence, six level A IVIVCs were established for these conditions and they are given in figures 5 and 6 . Three level A IVIVCs were established for the in vivo clinical trial performed under fasting condition, while 3 level $A$ IVIVCs were determined for the in vivo clinical trial carried out under the fed condition. 
Even though the established correlations were not meant to be scaledup at industrial level, the preliminary results of this study are promising and could be a good predictor of a possible IVIVC to be further developed in order to apply for a biowaiver. Thus, the costs of the manufacturing company for additional studies consecutive to post-approval changes would be lower as the drug product could benefit from a biowaiver given by the regulatory authorities on the basis of a level A IVIVC.

\section{CONCLUSIONS}

Six level A IVIVCs were established for gliclazide $60 \mathrm{mg}$ modified release tablets. The in vivo studies were two bioequivalence studies for newly developed gliclazide $60 \mathrm{mg}$ modified release tablet, under fasting and fed conditions. The in vitro studies were carried out in three different dissolution media, at $\mathrm{pH} 4.5,6.8$ and 7.2. The calculated point-to-point IVIVCs $\left(\mathrm{R}^{2}>0.98\right)$ could be further used for developing IVIVCs at industrial level which are useful from a regulatory point of view. The knowledge of the BCS class of a drug substance is essential for biowaivers, thus reducing the costs in terms of both time and money. Gliclazide belongs to class II of the BCS, characterized by low solubility and high permeability, thus level A IVIVCs were possible to establish with the in vivo data from the clinical trial of the new generic Gliclazide $60 \mathrm{mg}$ modified release formulation, developed by Ranbaxy Laboratories Limited, now Sun Pharmaceutical Industries Limited, India.

\section{EXPERIMENTAL SECTION}

\section{In vivo data}

The clinical study data (plasma concentration versus time profile) were obtained from two bioequivalence trials that took place at the Clinical Unit of Clinical Pharmacology and Pharmacokinetics Department of Terapia S.A. The study protocols were approved by the Ethics Committee of the University of Medicine and Pharmacy "Iuliu Hatieganu", from Cluj-Napoca (Romania) and by the National Agency for Medicines and Medical Devices, Romania. The first study took place under the fasting state and the second clinical trial was carried out under the fed state $[14,15]$. The clinical trials were conducted in accordance with all applicable regulatory requirements. These data were further used for in vitro-in vivo correlation purpose. 


\section{In vitro studies}

Dissolution studies were performed on $60 \mathrm{mg}$ generic gliclazide modified release tablets (EvoluPharm, France). The dissolution test was carried out in a rotating paddle apparatus (USP apparatus II) (Pharma Test, type PT WS100S, serial number 20208, D-63512 Hainburg, Germany) at $37 \pm 0.5^{\circ} \mathrm{C}$ and rotational speed of $50 \mathrm{rpm}$, using $900 \mathrm{ml}$ of various dissolution media (media pH 4.5, 6.8 and 7.2).

The dissolution media were prepared as it follows:

- $\mathrm{pH} 4.5$ sodium acetate buffer - $2.99 \mathrm{~g}$ of sodium acetate $\left(\mathrm{NaC}_{2} \mathrm{H}_{3} \mathrm{O}_{2}{ }^{*} 3 \mathrm{H}_{2} \mathrm{O}\right)$ were weighted and transferred in a volumetric flask of $1000 \mathrm{ml}, 14 \mathrm{ml}$ of $2 \mathrm{~N}$ acetic acid $\left(\mathrm{CH}_{3} \mathrm{COOH}\right)$ were added and then distilled water was used to made up to volume.

- $\mathrm{pH} 6.8$ phosphate buffer $-250 \mathrm{ml}$ of $0.2 \mathrm{M}$ monobasic potassium phosphate solution were transferred in a volumetric flask of $1000 \mathrm{ml}$, along with $112 \mathrm{ml}$ of $0.2 \mathrm{M} \mathrm{NaOH}$ and then distilled water was added to the volume.

- $\mathrm{pH} 7.4$ phosphate buffer $-250 \mathrm{ml}$ of $0.2 \mathrm{M}$ monobasic potassium phosphate solution were transferred in a $1000 \mathrm{ml}$ volumetric flask, then 195.5 $\mathrm{ml}$ of $0.2 \mathrm{M} \mathrm{NaOH}$ were added and made up to specified volume with distilled water.

All the reagents used for the preparation of the dissolution media buffer were of United States Pharmacopoeia (USP) reagents grade.

The samples $(100 \mu \mathrm{l}$ each) were taken before the release of the tablets in the dissolution media (time 0 ) and after, at the following times: 1 , $2,3,4,5,6,7,8,9,10,12,14,16,24$ hours. Withdrawn samples were filtered and assayed for gliclazide content by means of a validated HPLC UV bioanalytical method with detection at $225 \mathrm{~nm}$.

\section{In vitro - in vivo correlation}

In order to analyze the level of correlation, the numerical deconvolution method was used. In this approach, the absorption profile was obtained, based on the in vivo data from the clinical trials conducted under fasting and fed state of the subjects. Afterwards, the percent of drug absorbed in vivo was plotted against the dissolved percent in in vitro test [11]. Taking into account the time discrepancies between the in vivo absorption profiles and the in vitro release profiles for Gliclazide $60 \mathrm{mg}$ modified release tablets, a time scaling factor was considered for the determination of an IVIVC. 


\section{ACKNOWLEDGMENTS}

Financial disclosures: Ana-Maria Gheldiu and Laurian Vlase are full-time employees of the University of Medicine and Pharmacy "Iuliu Hatieganu”, Cluj-Napoca, Romania.

Conflicts of interest: Diana Pop, Adriana Marcovici, Monica Oroian were employees of the Ranbaxy Laboratories Limited, now Sun Pharmaceutical Industries, India, during the conduct of this study.

\section{REFERENCES}

1. G.L. Amidon, H. Lennernas, V.P. Shah, J.R. Crison, Pharmaceutical Research, 1995, 12(3), 413.

2. B.M. Davit, I. Kanfer, Y.C. Tsang, J.M. Cardot, The AAPS Journal, 2016, 18(3), 612.

3. L. Shargel, A.B.C. Yu, "Applied biopharmaceutics and pharmacokinetics, $7^{\text {th }}$ edition", McGraw-Hill Education, Inc., Maryland, 2016, chapter 15.

4. International Council for Harmonization of technical; requirements for pharmaceuticals for human use. Biopharmaceutics classification system-based biowaivers. 2018. Available at:

https://www.ich.org/fileadmin/Public_Web_Site/ICH_Products/Guidelines/Multi disciplinary/M9/M9EWG_DraftGuidēine_Step2_2018_0606.pdf. Accessed January 10, 2019.

5. J.E. Polli, The AAPS Journal, 2008, 10(2), 289.

6. S. Grbic, J. Parojcic, S. Ibric, Z. Djuric, AAPS PharmSciTech, 2011, 12(1), 165.

7. U.S. Department of Health and Human Services, Food and Drug Administration, Center for Drug Evaluation and Research. Guidance for Industry. Extended Release Oral Dosage Forms: Development, Evaluation, and Application of In Vitro/In Vivo Correlations. 1997. Available at:

https://www.fda.gov/downloads/drugs/guidances/ucm070239.pdf. Accessed January 10, 2019.

8. U.S. Department of Health and Human Services, Food and Drug Administration, Center for Drug Evaluation and Research. Guidance for Industry. SUPAC-MR: Modified Release Solid Oral Dosage Forms. 1997. Available at: https://www.fda.gov/downloads/Drugs/Guidances/ ucm070640.pdf. Accessed January 10, 2019.

9. U.S. Department of Health and Human Services, Food and Drug Administration, Center for Drug Evaluation and Research. Guidance for Industry. Waiver of In Vivo Bioavailability and Bioequivalence Studies for Immediate-Release Solid Oral Dosage Forms Based on a Biopharmaceutics Classification System. 2017. Available at: https://www.fda.gov/downloads/ Drugs/Guidances/ucm070246.pdf. Accessed January 10, 2019. 
10. L.X. Yu, G.L. Amidon, J.E. Polli et al., Pharmaceutical Research, 2002, 19(7), 921.

11. D.I. Pop, A.M. Gheldiu, M. Oroian, A. Marcovici, S. Bhardwaj, A. Khuroo, R. Kochhar, L. Vlase, Acta Medica Marisiensis, 2018, 64(4), 161.

12. M.B.V. Priya, T.E.G.K. Murthy, Dissolution Technologies, May 2012, 38.

13. K.K.S. Skripnik, M.K. Riekes, B.R. Pezzini, S.G. Cardoso, H.K. Stulzer, AAPS PharmSciTech, 2017, 18(5), 1785.

14. D.I. Pop, M. Oroian, S. Bhardwaj, A. Marcovici, A. Khuroo, R. Kochhar, L. Vlase, Clinical Pharmacology in Drug Development, 2019, 8(1), 16.

15. D.I. Pop, M. Oroian, S. Bhardwaj, A. Marcovici, A. Khuroo, R. Kochhar, L. Vlase, Farmacia, 2018, 66(4), 597.

16. The United States Pharmacopeia, $23^{\text {rd }}$ edition. "In Vitro and In Vivo Evaluation of Dosage form < 1088>". Rockville, Maryland, 1995, 1824.

17. J.M. Cardot, B.M. Davit, The AAPS Journal, 2012, 14(3), 491.

18. A. Sarkar, A. Tiwari, P.S. Bhasin, M. Mitra, Journal of Applied Pharmaceutical Science, 2011, 01(09), 11.

19. Summary of product characteristics Gliclazida ${ }^{\circledR}$ Terapia $60 \mathrm{mg}$. Available at: https://www.anm.ro/_/RCP/RCP_8149_30.09.15.pdf. Accessed January 10, 2019.

20. Product information Diamicron ${ }^{\circledR}$ MR $30 \mathrm{mg}$. Available at https://www.medicines.org.uk/emc/product/1321/smpc. Accessed January 10, 2019.

21. A Gumieniczek, A Berecka, R Pietras, M Slebioda, Cent Eur J Chem, 2014, 12(1), 80.

22. Chemical structure of gliclazide, available at: https://pubchem.ncbi.nlm.nih.gov/compound/gliclazide\#section=2D-Structure. Accessed on the $22^{\text {nd }}$ of May 2019. 
